\title{
Immunohistochemistry study of tumor vascular normalization and anti-angiogenic effects of sunitinib versus bevacizumab prior to dose-dense doxorubicin/cyclophosphamide chemotherapy in HER2-negative breast cancer
}

\author{
Kritika Yadav ${ }^{1,2}$. Joline Lim ${ }^{3}$. Joan $\mathrm{Choo}^{3}$. Samuel Guan Wei Ow ${ }^{3}$. Andrea Wong ${ }^{3}$ Matilda Lee ${ }^{3}$. Ching Wan Chan ${ }^{4}$. \\ Mikael Hartman ${ }^{4}$. Siew Eng Lim ${ }^{3}$. Natalie $\mathrm{Ngoi}^{3} \cdot$ Siau Wei Tang ${ }^{4} \cdot{\text { Yvonne } \mathrm{Ang}^{3} \text {. Gloria Chan }}^{3} \cdot$ Wan Qin Chong ${ }^{3}$. \\ Hon Lyn $\operatorname{Tan}^{3}$. Sing Huang $\operatorname{Tan}^{3} \cdot$ Boon Cher Goh ${ }^{2,3} \cdot$ Soo Chin Lee ${ }^{2,3}$
}

Received: 16 August 2021 / Accepted: 30 November 2021 / Published online: 20 December 2021

(c) The Author(s) 2021

\begin{abstract}
Purpose Tumor angiogenesis controlled predominantly by vascular endothelial growth factor and its receptor (VEGFVEGFR) interaction plays a key role in the growth and propagation of cancer cells. However, the newly formed network of blood vessels is disorganized and leaky. Pre-treatment with anti-angiogenic agents can "normalize" the tumor vasculature allowing effective intra-tumoral delivery of standard chemotherapy. Immunohistochemistry (IHC) analysis was applied to investigate and compare the vascular normalization and anti-angiogenic effects of two commonly used anti-angiogenic agents, Sunitinib and Bevacizumab, administered prior to chemotherapy in HER2-negative breast cancer patients.

Methods This prospective clinical trial enrolled 38 patients into a sunitinib cohort and 24 into a bevacizumab cohort. All received 4 cycles of doxorubicin/cyclophosphamide chemotherapy and pre-treatment with either sunitinib or bevacizumab. Tumor biopsies were obtained at baseline, after cycle 1 (C1) and cycle 4 (C4) of chemotherapy. IHC was performed to assess the tumor vascular normalization index (VNI), lymphatic vessel density (LVD), Ki67 proliferation index and expression of tumor VEGFR2.

Results In comparison to Bevacizumab, Sunitinib led to a significant increase in VNI post-C1 and C4 $(p<0.001$ and 0.001$)$ along with decrease in LVD post-C1 $(p=0.017)$. Both drugs when combined with chemotherapy resulted in significant decline in tumor proliferation after $\mathrm{C} 1$ and $\mathrm{C} 4$ (baseline vs post-C4 Ki67 index $p=0.006$ for Sunitinib vs $p=0.021$ for Bevacizumab). Bevacizumab resulted in a significant decrease in VEGFR2 expression post-C1 $(p=0.004)$.

Conclusion Sunitinib, in comparison to Bevacizumab showed a greater effect on tumor vessel modulation and lymphangiogenesis suggesting that its administration prior to chemotherapy might result in improved drug delivery.

Trial registry ClinicalTrials.gov: NCT02790580 (first posted June 6, 2016).
\end{abstract}

Keywords Anti-angiogenic $\cdot$ Bevacizumab $\cdot$ HER2-negative breast cancer $\cdot$ Sunitinib $\cdot$ Vascular normalization

Soo Chin Lee

csilsc@nus.edu.sg

1 Present Address: Department of Pathology, Dr. D Y Patil Medical College, Navi Mumbai, India

2 Cancer Science Institute, National University of Singapore, Singapore, Singapore

3 Department of Haematology-Oncology, National University Cancer Institute, National University Health System, Singapore, Singapore

4 Department of Surgery, National University Cancer Institute, National University Health System, Singapore, Singapore

\section{Introduction}

As per global cancer statistics 2018, breast cancer is the most commonly diagnosed cancer in females, with an incidence rate of 46.2 per 100,000 and representing $24.2 \%$ of the total female cancer burden globally. It is also the leading cause of cancer death with a mortality rate of $15 \%$ [1]. Multiple studies have highlighted the key role of tumor vascularization in facilitating tumor growth, progression and metastasis of various solid tumors including breast cancer $[2,3]$. Bevacizumab is an anti-vascular endothelial growth 
factor (VEGF) monoclonal antibody, while Sunitinib is an orally administered small molecule receptor tyrosine kinase inhibitor that exerts its action by targeting the vascular endothelial growth factor receptors (VEGFR). Both agents have been studied in clinical trials in combination with chemotherapy in breast cancer. Disappointingly, although both anti-angiogenic agents have shown promising preclinical results, their effects in breast cancer when combined with chemotherapy have been conflicting in the clinic. This could be in part attributed to the fact that optimal dosing schedule of these drugs in combination with chemotherapy are yet to be determined [4-6].

A major pathway involved in angiogenesis is the release of VEGF from hypoxic tumor cells and its binding to the VEGFR expressed on the vascular and lymphatic endothelial cells, leading to endothelial cell proliferation and migration. However, the newly formed blood vessels in a growing tumor are dilated, leaky and poorly organized with no pericyte covering. These "immature" vessels have variable blood flow resulting in sub-optimal delivery of chemotherapeutic drugs. The careful and judicious use of anti-angiogenic drugs can "normalize" these abnormally structured blood vessels within the tumor leading to more efficient drug delivery [7]. In addition, VEGFR2 is expressed on various tumors including breast cancer and is responsible for the autocrine and paracrine effect of VEGF resulting in tumor cell survival and proliferation [8].

We hypothesize that pre-treatment rather than concurrent treatment with sunitinib or bevacizumab prior to standard chemotherapy in human epidermal growth factor receptor 2 (HER2)-negative breast cancer, along with a lower dose of the anti-angiogenic agent, will improve and "normalize" the tumor vasculature making it more efficient for intra-tumoral chemotherapy drug delivery. We enrolled patients into a prospective clinical trial and obtained serial tumor biopsies at baseline, during and after chemotherapy to assess and compare the vascular normalization and anti-angiogenic effects of sunitinib versus bevacizumab. Vascular normalization, lymphatic density, tumor proliferation index and activated VEGFR2 status of tumor cells were studied in the tumor specimens.

\section{Patients and methods}

\section{Study population}

Patients were enrolled into a prospective, phase II open label, single-arm study conducted at the National University Cancer Institute, Singapore (NCIS). Eligibility criteria included female patients aged $\geq 18$ years with newly diagnosed and histologically confirmed HER2-negative breast cancer. HER 2 negativity was defined as HER 2 score 0 or
$1+$ on immunohistochemistry (IHC) or HER 2 IHC $2+$ but HER2 Fluorescence In Situ Hybridization (FISH)-negative (HER2/CEP17 ratio $<2.0$ with gene copy number $<4.0$ signals/ cell) [9]. Other inclusion criteria were measurable primary tumor $\geq 2 \mathrm{~cm}$, Eastern Cooperative Oncology Group $(E C O G)$ performance 0 or 1, absolute neutrophilic count $\geq 1.5 \times 10^{9} / \mathrm{L}$, platelets $\geq 100 \times 10^{9} / \mathrm{L}$, serum total bilirubin $\leq 1.5 \times$ upper limit of normal (ULN), alanine and aspartate aminotransferase $\leq 2.5 \times \mathrm{ULN}$, serum creatinine $\leq 1.5 \times \mathrm{ULN}$ and left ventricular ejection fraction $\geq 50 \%$. Patients with clinically detectable second primary malignancy, symptomatic brain metastasis, and known history of systemic connective tissue diseases were excluded from the study. Signed written informed consent was taken from all patients before enrollment. The clinical trial was conducted in accordance with local regulatory requirements and approved by the institutional ethics review board.

\section{Treatment plan and study design}

The study aimed to determine the effect of pre-treatment with low-dose anti-angiogenic agent prior to chemotherapy as a strategy to normalize tumor vasculature. Patients were enrolled into two sequential Cohorts to study two different classes of anti-angiogenic agents: Sunitinib Cohort to evaluate a small molecule tyrosine kinase inhibitor against VEGFR, and Bevacizumab Cohort to evaluate a monoclonal antibody against VEGF. All subjects received 4 cycles of dose-dense doxorubicin/cyclophosphamide (ddAC) chemotherapy every 2 weeks (doxorubicin $60 \mathrm{mg} / \mathrm{m}^{2}$ and cyclophosphamide $600 \mathrm{mg} / \mathrm{m}^{2}$ ) supported by prophylactic pegfilgrastim $6 \mathrm{mg}$ administered subcutaneously $24-48 \mathrm{~h}$ after each cycle of chemotherapy. Patients enrolled into the Sunitinib Cohort were pre-treated with oral sunitinib $12.5 \mathrm{mg}$ daily for 7 days prior to cycle $1 \mathrm{ddAC}$ and for 5 days prior to cycles 2, 3 and 4 ddAC. Patients enrolled into the Bevacizumab Cohort received intravenous bevacizumab $5 \mathrm{mg} / \mathrm{kg}$, 7 days prior to each chemotherapy cycle. Dosing of sunitinib was determined through a phase Ib/II study by Wong et al. and dosing of bevacizumab was determined based on extrapolation from a preclinical study by Gaustad et al. [10, 11]. Primary endpoint of the study was rate of pathological complete response ( $\mathrm{pCR}$ ) post neoadjuvant chemotherapy, defined as absence of invasive cancer in both breast and axillary lymph nodes time of surgery, with pharmacodynamic biomarker exploration through IHC as a secondary endpoint.

For each patient, detailed history was recorded. Physical examination, radiological staging with computed tomography (CT) scan or CT-PET scan and laboratory evaluation was done prior to initiating treatment. Patients were evaluated before each new cycle of chemotherapy to monitor adverse effects from treatment and to measure tumor response. Response to treatment was assessed clinically 
according to the Response Evaluation Criteria in Solid Tumors (RECIST) version 1.1 criteria [12].

\section{Tumor core biopsies for immunohistochemistry (IHC) studies}

Tumor core biopsies were obtained from the primary breast tumor under ultrasound guidance at baseline, 2 weeks after cycle $1 \mathrm{ddAC}$ but before cycle $2 \mathrm{ddAC}$ (post-C1) and about 2 weeks after completion of 4 cycles of ddAC (post-C4). Tumor cores were fixed in formalin for further histological and IHC analysis.

\section{Immunohistochemistry studies}

Tumor biopsies taken at each time point were processed into paraffin blocks. Hematoxylin and Eosin (H\&E) staining was done to identify and assess the tumor content. Biopsies which showed tumor content $\leq 10 \%$ were excluded from further evaluation and staining. IHC was performed on consecutive slides using the Leica Bond Max automated platform (Leica Biosystems, Nussloch $\mathrm{GmbH}$ ) with bond polymer refine detection kit and bond polymer refine red detection kit (DS9800 and DS9390, respectively, Leica Biosystems). Briefly, 4-micron sections from tissue blocks were taken on coated slides. These were then deparaffinized, hydrated and blocked with hydrogen peroxide. Heat induced antigen retrieval was achieved using appropriate buffer for each antibody as per optimized protocol in control tissue. Slides were incubated with primary antibody followed by secondary antibody. Staining was completed with diaminobenzidine (DAB) chromogen and haematoxylin was used as a counterstain.

To assess the tumor vascular normalization index (VNI), double sequential staining for endothelial cells and pericytes was performed using CD31 and alpha-smooth muscle actin ( $\alpha$-SMA), respectively. This was followed by visualization with alkaline phosphatase-based red (DS9390) and peroxidase-based diaminobenzidine polymer detection systems (DS9800), respectively. VNI was calculated as the percentage of CD31-positive cells which co-express $\alpha$-SMA in relation to the total number of blood vessels in the entire biopsy. This index was used as an indicator of tumor vessel maturation [13]. D2-40 antibody was selected for marking the lymphatic vessels and analyzing the lymphatic vessel density (average number of vessels positive for D2-40 in the entire biopsy). To study the pharmacodynamic effect of sunitinib and bevacizumab on tumor cells, the expression of activated VEGFR2 (phosphorylated VEGFR2 at tyrosine phosphorylation sites 951 and 996 [Y951 and Y996]) on tumor cells was examined. VEGFR2 expression was semiquantified by $\mathrm{H}$ score, which is the percentage of tumor cells staining positive multiplied by an intensity score $(0$ : no staining, 1: weak staining, 2: moderate staining, 3: strong staining). The final score ranged from 0 to 300 [14]. Ki67 proliferation index of tumor was calculated as percentage of positively stained cells among the total number of malignant cells [15]. Details on the antibody clone, commercial supplier, dilution and antigen retrieval are provided in Table 1.

\section{Evaluation of histological response on surgical specimens}

After completing 4 cycles of ddAC chemotherapy, patients with non-metastatic cancer underwent lumpectomy or mastectomy and sentinel lymph node biopsy or axillary lymph node clearance. Scoring of histological response on the primary tumor was done using the 5-point scale Miller-Payne grading (MPG) classification which was based on comparing the tumor cellularity between baseline and post-C4 biopsy. Good histological response was defined as a score of $\geq 3$, i.e., more than $30 \%$ reduction of tumor cellularity in posttreatment biopsies from baseline [16].

\section{Statistical analysis}

All statistical analyses were performed using SPSS software version 20.0 (IBM, Armonk, NY, USA). Categorical variables were presented as frequencies and percentages, while continuous variables as mean with standard deviation (SD). Comparison of continuous data between groups was done using the non-parametric Wilcoxon signed rank test. Categorical data were compared using Chi-square test. Independent $t$-test was run for comparison of means. A p value of less than 0.05 was considered statistically significant.
Table 1 Details of antibodies used in immunohistochemistry studies

\begin{tabular}{lllll}
\hline Antibody & Clone & Manufacturer & Dilution & Antigen retrieval \\
\hline CD31 & JC70A & Dako & $1: 100$ & Citrate buffer, pH 6, 20 min \\
o-SMA & 1A4 & Dako & $1: 500$ & Citrate buffer, pH 6, 20 min \\
D2-40/podoplanin & D2-40 & Dako & $1: 100$ & Citrate buffer, pH 6, 20 min \\
p-VEGFR2 951 & Rabbit polyclonal & Invitrogen & $1: 50$ & Citrate buffer, pH 6, 20 min \\
p-VEGFR2 996 & Rabbit polyclonal & Invitrogen & $1: 100$ & EDTA buffer, pH 9, 20 min \\
Ki67 & MIB-1 & Dako & $1: 100$ & EDTA buffer, pH 9, 20 min \\
\hline
\end{tabular}




\section{Results}

A total of sixty-two subjects were recruited; 38 patients were enrolled into the Sunitinib Cohort and 24 into the Bevacizumab Cohort. The baseline demographic and tumor characteristics of the patients are summarized in Table 2. Median age of the entire cohort was 51 years (range 29-70). Majority of the patients were Chinese, had invasive ductal carcinoma, hormone receptor-positive cancer, and non-metastatic disease. There were no significant differences in demographic or baseline tumor characteristics between the Sunitinib and Bevacizumab Cohorts.

\section{Immunohistochemistry studies}

Out of the total 62 patients, a full set of pre-treatment, post-C1 and post-C4 tumor samples were available for IHC analysis for 29 of the 38 patients enrolled into the sunitinib cohort, and 15 of the 24 patients enrolled into the Bevacizumab cohort. An additional 5 patients enrolled into the bevacizumab cohort had pre-treatment and post-C1 samples without a post-C4 tumor sample. Reasons for incomplete tumor specimens for IHC analysis include no biopsy because of complete clinical response, core biopsy tumor specimen too small, or insufficient tumor content for IHC analysis (Fig. 1).
Table 2 Baseline clinical and pathological characteristics of patients in the two treatment groups

\begin{tabular}{|c|c|c|c|}
\hline \multirow[t]{2}{*}{ Characteristics/Variables } & \multicolumn{3}{|c|}{ Number (percentage) } \\
\hline & Sunitinib $(n=38)$ & $\begin{array}{l}\text { Bevacizumab } \\
(n=24)\end{array}$ & $p$ value \\
\hline \multicolumn{4}{|l|}{ Age (years) } \\
\hline Median & 53.5 & 49.5 & \multirow[t]{2}{*}{0.406} \\
\hline Range & $30-69$ & $29-70$ & \\
\hline \multicolumn{4}{|l|}{ Race } \\
\hline Chinese & $24(63.1)$ & $16(66.7)$ & \multirow[t]{4}{*}{0.679} \\
\hline Malay & $5(13.2)$ & $5(20.8)$ & \\
\hline Indian & $3(7.9)$ & $1(4.2)$ & \\
\hline Others & $6(15.8)$ & $2(8.3)$ & \\
\hline \multicolumn{4}{|l|}{ Histological type of tumor } \\
\hline Ductal & $33(86.8)$ & $20(83.3)$ & \multirow[t]{3}{*}{0.887} \\
\hline Lobular & $3(7.9)$ & $2(8.3)$ & \\
\hline Others & $2(5.3)$ & $2(8.3)$ & \\
\hline \multicolumn{4}{|l|}{ Histological grade of tumor } \\
\hline Grade 1 (Well differentiated) & $2(5.3)$ & 0 & \multirow[t]{3}{*}{0.183} \\
\hline Grade 2 (Moderately differentiated) & $10(26.3)$ & $11(45.8)$ & \\
\hline Grade 3 (poorly differentiated) & $26(68.4)$ & $13(54.2)$ & \\
\hline \multicolumn{4}{|l|}{ Hormone receptor status } \\
\hline ER and/or PR-Positive & $29(76.3)$ & $18(75)$ & \multirow[t]{2}{*}{0.906} \\
\hline ER/PR-Negative & $9(23.7)$ & $6(25)$ & \\
\hline \multicolumn{4}{|l|}{ Clinical T stage of primary tumor } \\
\hline $\mathrm{T} 1$ & $1(2.6)$ & $1(2.6)$ & \multirow[t]{4}{*}{0.988} \\
\hline $\mathrm{T} 2$ & $21(55.3)$ & $13(34.2)$ & \\
\hline T3 & $10(26.3)$ & $6(15.8)$ & \\
\hline $\mathrm{T} 4$ & $6(15.8)$ & $4(10.5)$ & \\
\hline \multicolumn{4}{|l|}{ Clinical node status } \\
\hline N0 & $10(26.3)$ & $10(41.6)$ & \multirow[t]{4}{*}{0.569} \\
\hline N1 & $22(57.9)$ & $12(50)$ & \\
\hline $\mathrm{N} 2$ & $2(5.3)$ & $1(4.2)$ & \\
\hline N3 & $4(10.5)$ & $1(4.2)$ & \\
\hline \multicolumn{4}{|l|}{ Metastasis } \\
\hline Present & $5(13.2)$ & $2(8.3)$ & \multirow[t]{2}{*}{0.559} \\
\hline Absent & $33(86.8)$ & $22(91.7)$ & \\
\hline
\end{tabular}

$E R$ estrogen receptor, $P R$ progesterone receptor 


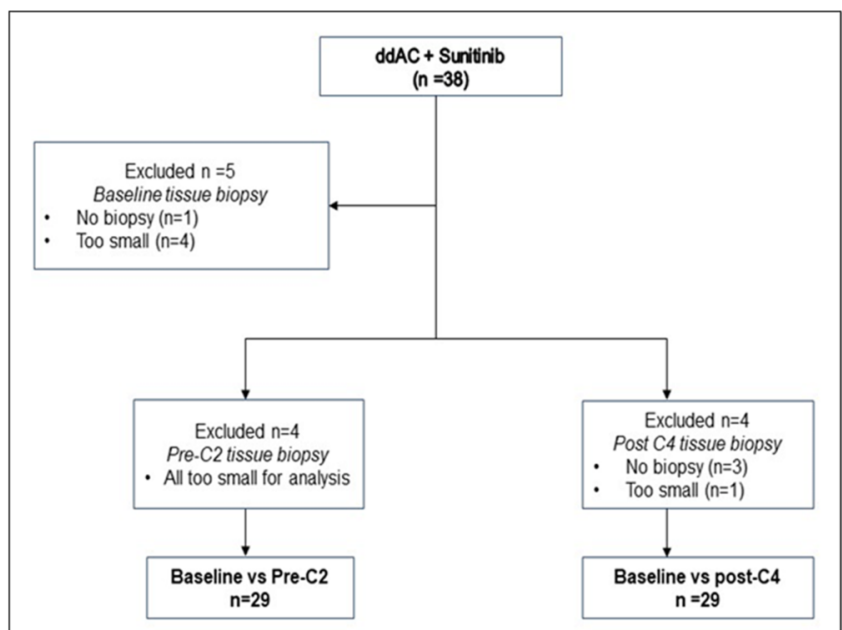

Fig. 1 Consort diagram showing the selection of cases for immunohistochemical analysis. No biopsy-Surgical biopsy not taken at that timepoint, Too small-Tissue was very small for multiple sections

\section{Changes in lymphatic vessel density, vascular normalization index, Ki67 and p-VEGFR2 induced by Sunitinib versus Bevacizumab}

In the Sunitinib Cohort, there was a significant decrease in tumor lymphatic vessel density (LVD) after cycle 1 that persisted after cycle 4 chemotherapy compared to baseline (mean LVD $0.94 \pm 1.39,0.29 \pm 0.45,0.36 \pm 0.58$ for

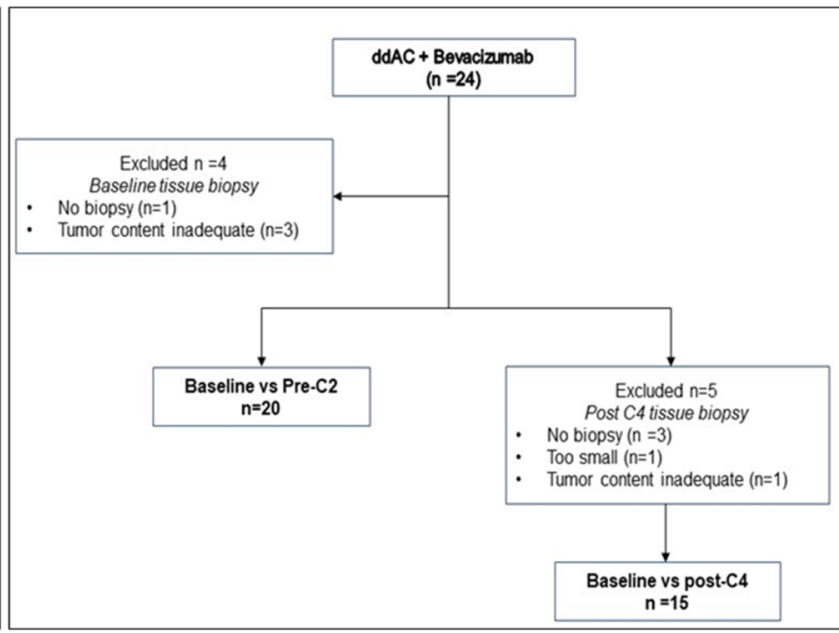

required for histological and immunohistochemistry analysis, Inadequate-Biopsies which showed tumor content $\leq 10 \%$

baseline, post-C1 and post-C4; $p=0.017$ for baseline vs post-C1, $p=0.112$ for baseline vs post-C4) (Fig. 2a, b). In contrast, there was a numerical increase in LVD that was not statistically significant after cycle 1 or cycle 4 chemotherapy compared to baseline observed in the Bevacizumab Cohort (Table \& Fig. 3). While there were no differences in LVD at baseline among sunitinib-treated patients by clinical or pathological nodal status, there was a trend that those

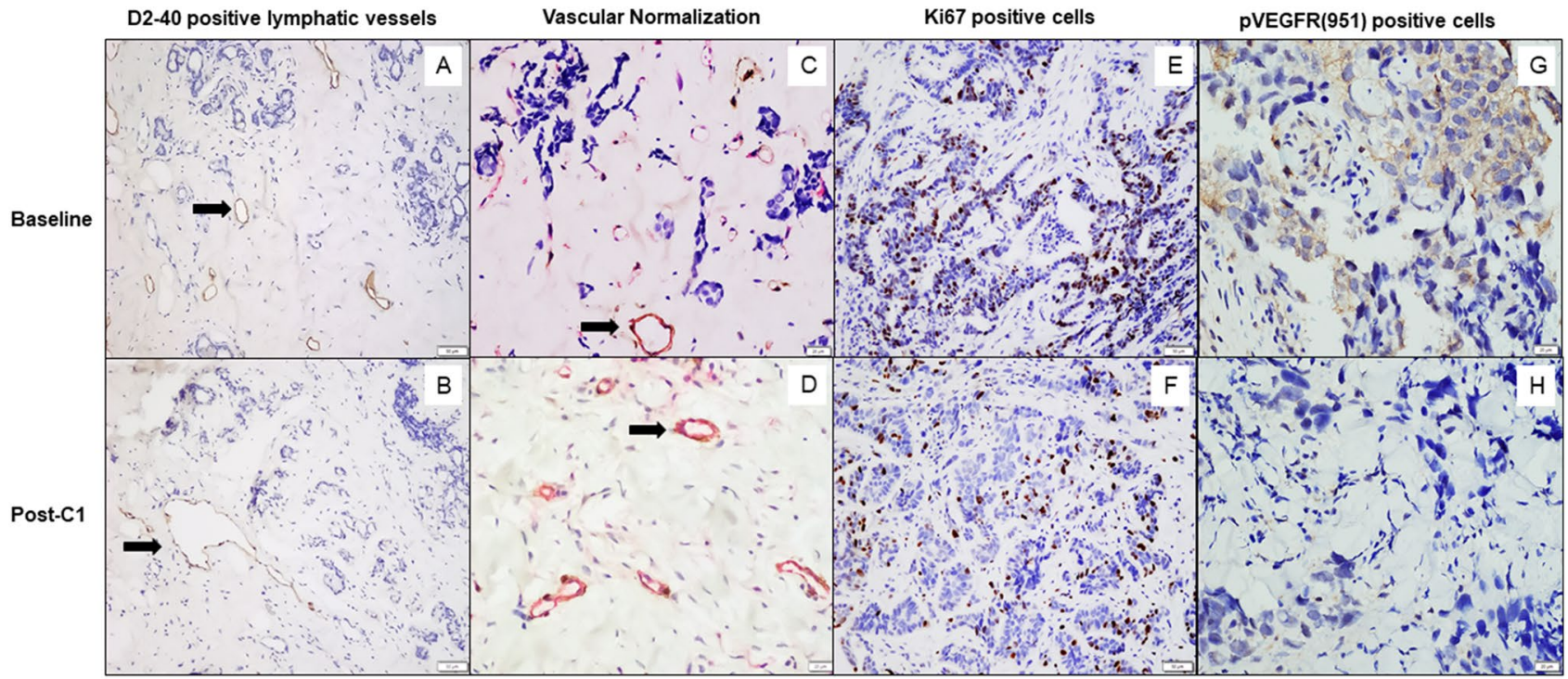

Fig. 2 In a representative biopsy from a patient pre-treated with sunitinib, immunohistochemistry staining showing decrease in the number of D2-40-positive lymphatic vessels post-cycle 1 compared to baseline (Image $\mathbf{a}$ and $\mathbf{b} ; \times 200$ magnification). Increase in vascular normalization index seen in post-cycle 1 where CD31+vessels (in red) are covered with a layer of SMA + pericytes (in brown), in com- parison to baseline (Image $\mathbf{c}$ and $\mathbf{d} ; \times 400 \mathrm{X}$ magnification). Reduction of Ki67 proliferation index in tumor cells post-cycle 1 compared to baseline (Image $\mathbf{e}$ and $\mathbf{f} ; \times 200$ magnification). Decrease in expression of p-VEGFR2 (Y951) in tumor cells post-cycle 1 in comparison to baseline observed in a representative biopsy from patient pretreated with bevacizumab (Image $\mathbf{g}$ and $\mathbf{h} ; \times 400$ magnification) 


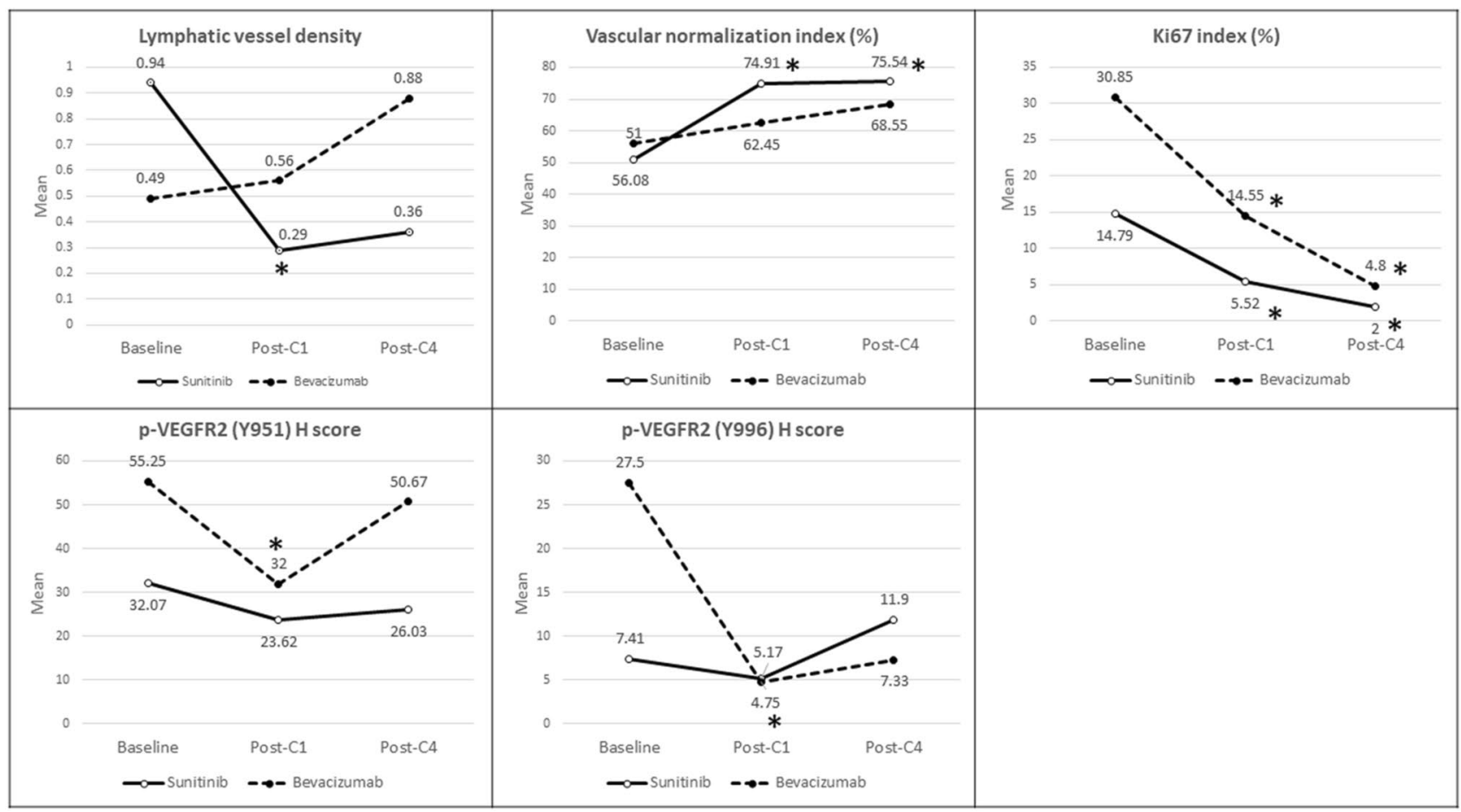

Fig. 3 Graphical representation comparing the change in IHC parameters at cycle 1 and cycle 4 from baseline, between the two cohorts. In contrast to Bevacizumab, Sunitinib cohort showed a significant decrease from baseline in lymphatic vessel density after cycle 1 along with a sustained significant increase in vascular normalization index after cycle 1 and cycle 4 . Bevacizumab cohort revealed a significant

who were pathologically node-negative after neoadjuvant chemotherapy had lower LVD post-cycle 1 treatment than those who were pathologically node-positive $(0.13 \pm 0.17 \mathrm{vs}$ $0.41 \pm 0.54, p=0.06$ ).

Significant increase in Vascular normalization Index (VNI) was observed in the Sunitinib Cohort after one cycle of chemotherapy and that persisted after four cycles of chemotherapy, compared to baseline (mean VNI $51.00 \pm 21.97 \%$, $74.91 \pm 18.93 \%, 75.54 \pm 21.23 \%$ for baseline, post-C1 and post-C4; $p<0.001$ for baseline vs post-C1, $p=0.001$ for baseline vs post-C4) (Fig. 2c, d). While a similar trend was observed in the Bevacizumab Cohort, the differences were not statistically significant (Table 3, Fig. 3).

In both the Sunitinib and Bevacizumab Cohorts, there was a significant decline in Ki67 proliferation index after cycle 1 and cycle 4 chemotherapy, although the decline appears quicker and more marked in the Sunitinib Cohort (mean Ki67 in Sunitinib Cohort $14.79 \pm 23.81 \%, 5.52 \pm 10.39$, $2.00 \pm 7.54 \%$ for baseline, post-C1 and post-C4; $p=0.027$ for baseline vs post-C1, $p=0.006$ for baseline vs postC4; mean Ki67 in Bevacizumab Cohort 30.85 \pm 33.16 , $14.55 \pm 22.96,4.80 \pm 8.26$ for baseline, post-C1 and post$\mathrm{C} 4 ; p=0.005$ for baseline vs post-C1, $p=0.021$ for baseline vs post-C4) (Fig. 2e, f) (Table 3, Fig. 3). decrease in p-VEGFR2 (Y951 and Y996) expression in tumor cells post-cycle 1 from baseline, indicating its inhibitory effect on tumor growth. Both cohorts display significant decline in Ki67 tumor proliferation index after cycle 1 and 4; however, the reduction appears earlier and more marked in the sunitinib cohort. *Significant change from baseline $(p<0.05)$

Expression of p-VEGFR2 on tumor cells was significantly reduced after cycle one chemotherapy in the Bevacizumab Cohort for both Y951 and Y996, although there appears to be a rebound in p-VEGFR2 after cycle 4 chemotherapy (mean $H$ score [Y951] 55.25 $\pm 41.15,32.00 \pm 38.60,50.67 \pm 41.82$ for baseline, post- $\mathrm{C} 1$ and post-C4; $p=0.008$ for baseline vs post-C1, $p=0.819$ for baseline vs post-C4; mean $\mathrm{H}$ score [Y996] $27.50 \pm 38.54,4.75 \pm 9.79,7.33 \pm 10.83$ for baseline, post-C 1 and post-C4; $p=0.004$ for baseline vs post$\mathrm{C} 1, p=0.124$ for baseline vs post-C4) (Fig. $2 \mathrm{~g}, \mathrm{~h}$ ). A similar trend was noted in the Sunitinib Cohort but the difference was not statistically significant (Table 3, Fig. 3).

\section{Clinical and pathological outcomes and correlation with IHC parameters}

After 4 cycles of ddAC chemotherapy, $2.7 \%$ and $8.3 \%$ of patients in the Sunitinib and Bevacizumab Cohorts respectively achieved complete clinical response; $73 \%$ and $75 \%$ respectively achieved clinical partial response, while $24.3 \%$ and $16.7 \%$ achieved stable disease. No patient in either Cohort had clinical progressive disease. Patients with a complete or partial response were considered as good clinical responders, while those with only stable disease as poor 


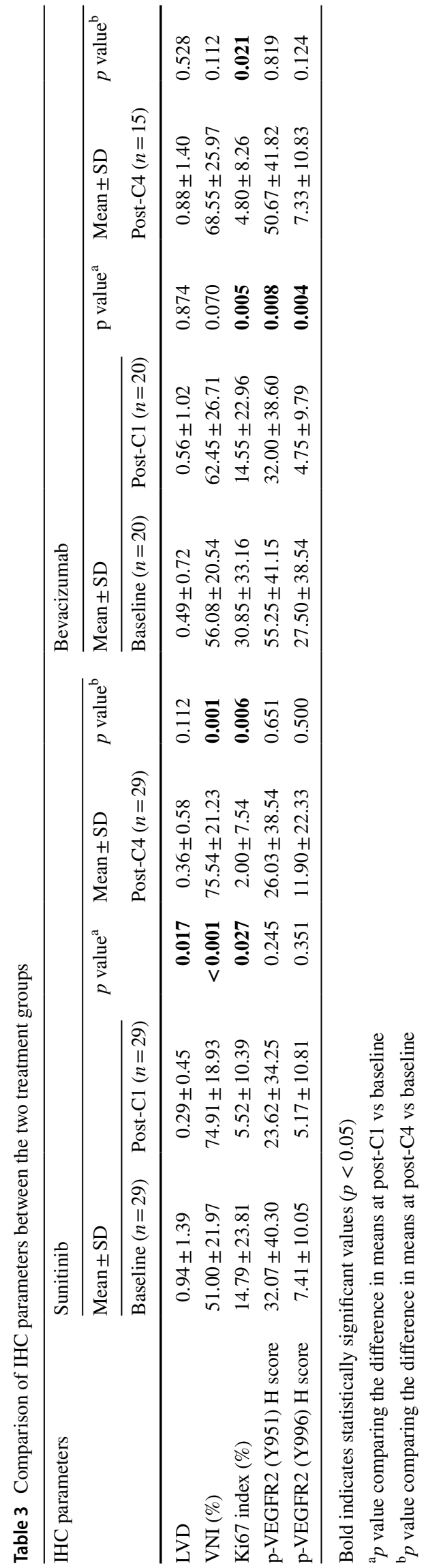

clinical responders. Thirty-three patients in the Sunitinib Cohort and 17 patients in the Bevacizumab Cohort underwent surgery. No patient achieved pathological complete response. Using the MPG system classification, $66.7 \%$ and $76.5 \%$ in the Sunitinib and Bevacizumab Cohorts respectively achieved good histological response after 4 cycles of ddAC. No significant differences in clinical or histological responses were observed between the Sunitinib and Bevacizumab Cohorts (percentage good clinical responders in Sunitinib vs Bevacizumab Cohorts $75.7 \%$ vs $83.3 \%$, $p=0.57$; percentage good histological responders in Sunitinib vs Bevacizumab Cohorts $66.7 \%$ vs $76.5 \%, p=0.47$ ). There was a statistically significant difference in the post-C4 Ki67 index between clinical good vs poor responders in both cohorts (Sunitinib cohort, mean Ki67 index 0.74 $\pm 2.07 \%$ vs $8.20 \pm 17.78 \%$ for good vs poor responders; $p=0.04$; Bevacizumab cohort, mean Ki67 index $3.15 \pm 4.75 \%$ vs $15.50 \pm 20.50 \%$ for good vs poor responders; $p=0.044$ ). However, there was no significant difference in the other IHC parameters (VNI, LVD, p-VEGFR2 H score) between good and poor clinical and histological responders in both the cohorts (Tables 4 and 5).

\section{Discussion}

Ever since the proposal of the "vascular normalization" theory, many preclinical observations show that the addition of anti-angiogenic agents to systemic chemotherapy leads to improved and uniform drug delivery and tumor control. The concept of presence of "normalization window" has provided insight into the probable benefit of administering low-dose and short-course anti-angiogenic drugs with chemotherapy instead of high and prolonged dosing $[17,18]$. However, limited clinical data is available to prove the same. In this study we performed an immunohistochemistry analysis to investigate and compare the effects of two anti-angiogenic drugs, sunitinib and bevacizumab, that are commonly used in clinical practice. Doses of sunitinib was administered at a third of FDA approved dosing, and bevacizumab dosing was also lower than that studied in previous breast cancer trials including the RIBBON1 study to achieve a "vascular normalization" effect rather than anti-angiogenic effect [19]. We examined vascular normalization, lymphatic vessel density, tumor proliferation index and activated VEGFR2 status of tumor cells in both treatment groups.

Tumor vasculature normalization has been observed as early as 7 days after treatment [10]. In our previous study (Wong et al.) evaluating low-dose, short-course sunitinib prior to chemotherapy, serial tumor biopsies and DCE-MRI at baseline, 7 days post-treatment and 28 days post-treatment in a small cohort of patients had shown the normalization effects to occur at 7 days but sustained at 28 days 
Table 4 Correlation of IHC parameters with good vs poor clinical response in the two treatment groups

\begin{tabular}{|c|c|c|c|c|c|c|}
\hline \multirow{3}{*}{$\begin{array}{l}\text { IHC parameters at } \\
\text { timepoints }\end{array}$} & \multicolumn{3}{|l|}{ Sunitinib $(n=28)^{\mathrm{a}}$} & \multicolumn{3}{|c|}{ Bevacizumab $(n=20)^{\mathrm{b}}$} \\
\hline & \multicolumn{3}{|l|}{ Mean \pm SD } & \multicolumn{3}{|l|}{ Mean \pm SD } \\
\hline & Good responder $(n=23)$ & Poor responder $(n=5)$ & $p$ value & $\begin{array}{l}\text { Good responder } \\
(n=16)^{\mathrm{b}}\end{array}$ & Poor responder $(n=4)^{b}$ & $p$ value \\
\hline \multicolumn{7}{|l|}{ Baseline } \\
\hline LVD & $0.83 \pm 1.29$ & $0.80 \pm 0.98$ & 0.97 & $0.42 \pm 0.51$ & $0.75 \pm 1.36$ & 0.43 \\
\hline VNI (\%) & $52.88 \pm 21.57$ & $40.58 \pm 25.37$ & 0.27 & $53.93 \pm 19.72$ & $64.65 \pm 24.59$ & 0.36 \\
\hline Ki67 index (\%) & $14.00 \pm 23.63$ & $21.20 \pm 28.19$ & 0.55 & $27.31 \pm 33.02$ & $44.00 \pm 38.73$ & 0.35 \\
\hline $\begin{array}{l}\text { p-VEGFR2 (Y951) H } \\
\text { score }\end{array}$ & $33.04 \pm 42.04$ & $26.00 \pm 39.74$ & 0.73 & $53.13 \pm 41.42$ & $63.75 \pm 44.97$ & 0.65 \\
\hline $\begin{array}{l}\text { p-VEGFR2 (Y996) H } \\
\text { score }\end{array}$ & $8.04 \pm 10.84$ & $6.00 \pm 6.51$ & 0.69 & $29.38 \pm 48.53$ & $20.00 \pm 16.33$ & 0.67 \\
\hline \multicolumn{7}{|l|}{ Post-C1 } \\
\hline LVD & $0.34 \pm 0.48$ & $0.12 \pm 0.16$ & 0.32 & $0.68 \pm 1.11$ & $0.05 \pm 0.010$ & 0.27 \\
\hline VNI (\%) & $74.61 \pm 20.18$ & $71.28 \pm 9.75$ & 0.72 & $66.92 \pm 23.60$ & $44.57 \pm 34.65$ & 0.13 \\
\hline Ki67 index (\%) & $4.70 \pm 10.13$ & $10.40 \pm 12.28$ & 0.28 & $10.61 \pm 19.71$ & $30.00 \pm 31.62$ & 0.13 \\
\hline $\begin{array}{l}\text { p-VEGFR2 (Y951) H } \\
\text { score }\end{array}$ & $27.61 \pm 36.30$ & $10.00 \pm 22.30$ & 0.31 & $25.00 \pm 34.83$ & $60.00 \pm 45.46$ & 0.1 \\
\hline $\begin{array}{l}\text { p-VEGFR2 (Y996) H } \\
\text { score }\end{array}$ & $5.65 \pm 11.90$ & $4.00 \pm 5.47$ & 0.76 & $5.94 \pm 10.68$ & 0 & 0.29 \\
\hline \multicolumn{7}{|l|}{$\begin{array}{l}\text { Post-C } 4^{\mathrm{b}}(n=15 \text { for } \\
\text { bevacizumab) }\end{array}$} \\
\hline LVD & $0.44 \pm 0.61$ & $0.08 \pm 0.09$ & 0.21 & $0.64 \pm 0.94$ & $2.4 \pm 3.39$ & 0.1 \\
\hline VNI (\%) & $78.84 \pm 16.06$ & $75.49 \pm 15.71$ & 0.67 & $70.63 \pm 23.92$ & $55.06 \pm 46.44$ & 0.45 \\
\hline Ki67 index (\%) & $0.74 \pm 2.07$ & $8.20 \pm 17.78$ & 0.04 & $3.15 \pm 4.75$ & $15.00 \pm 20.50$ & 0.04 \\
\hline $\begin{array}{l}\text { p-VEGFR2 (Y951) H } \\
\text { score }\end{array}$ & $27.17 \pm 41.22$ & $26.00 \pm 29.66$ & 0.95 & $43.08 \pm 39.45$ & $100.00 \pm 14.14$ & 0.71 \\
\hline $\begin{array}{l}\text { p-VEGFR2 (Y996) H } \\
\text { score }\end{array}$ & $10.43 \pm 19.93$ & $21.00 \pm 33.98$ & 0.35 & $6.92 \pm 10.90$ & $10.00 \pm 14.14$ & 0.72 \\
\hline
\end{tabular}

Bold indicates statistically significant values $(p<0.05)$

${ }^{\text {a }}$ Clinical response was not available for one patient of the total 29 patients whose biopsy samples were analyzed at all time points

${ }^{\mathrm{b}}$ In bevacizumab cohort, post-C4 biopsy IHC analysis was possible for 15 of total 20 patients. Of those 15 patients, 13 were good responders and 2 were poor responders

post-treatment [11]. As it is logistically challenging to perform multiple serial tumor biopsies on a larger cohort of patients, in this current trial, we have chosen to take the second biopsy 2 weeks after cycle 1 chemotherapy + antiangiogenic agent, which we believe is still early enough to observe normalization effects.

We demonstrated that in HER2-negative breast cancers, pre-treatment with low-dose short-course sunitinib leads to statistically significant increase in vascular normalization index in comparison to bevacizumab. Though both sunitinib and bevacizumab are capable of establishing a more mature vascular network in tumor microenvironment, sunitinib showed more promising results. Sunitinib led to almost $45 \%$ increase in VNI after one cycle of treatment compared to baseline although further increase from cycle 1 to cycle 4 was minor. On the other hand, pre-treatment with bevacizumab resulted in only $~ 10$ to $20 \%$ increase in VNI post-cycle 1 and 4 that was not statistically significant. The lymphatic vasculature also plays an important role in tumor cell progression and metastasis. Invasion in lymphatic vessels has been found to be associated with increased risk of lymph node and distant metastasis thereby leading to poor survival in breast cancer patients [20]. In our study, sunitinib appeared to inhibit lymphangiogenesis leading to significant decline in LVD after 1 cycle of treatment. In contrast, bevacizumab pre-treatment actually led to a numerical increase in LVD, albeit not statistically significant.

We previously conducted a phase $\mathrm{Ib} / \mathrm{II}$ trial in which subjects were randomized to chemotherapy with or without lowdose, intermittent sunitinib. IHC evaluation on serial tumor biopsies showed evidence of increased VNI and decrease in LVD after chemotherapy in patients randomized to receive sunitinib, but not in those treated with chemotherapy alone [11]. The observations in this current study in the Sunitinib 
Table 5 Correlation of IHC parameters with good vs poor histological response in the two treatment groups

\begin{tabular}{|c|c|c|c|c|c|c|}
\hline \multirow{3}{*}{$\begin{array}{l}\text { IHC parameters at time } \\
\text { points }\end{array}$} & \multicolumn{3}{|l|}{ Sunitinib $(n=26)^{\mathrm{a}}$} & \multicolumn{3}{|c|}{ Bevacizumab $(n=16)^{\mathrm{b}}$} \\
\hline & \multicolumn{3}{|l|}{ Mean \pm SD } & \multicolumn{3}{|l|}{ Mean \pm SD } \\
\hline & Good responder $(n=17)$ & Poor responder $(n=9)$ & $p$ value & $\begin{array}{l}\text { Good responder } \\
(n=12)^{\mathrm{b}}\end{array}$ & Poor responder $(n=4)^{\mathrm{b}}$ & $p$ value \\
\hline \multicolumn{7}{|l|}{ Baseline } \\
\hline LVD & $0.88 \pm 1.49$ & $0.68 \pm 0.83$ & 0.72 & $0.56 \pm 0.86$ & $0.20 \pm 0.28$ & 0.42 \\
\hline VNI & $56.18 \pm 19.49$ & $44.25 \pm 21.42$ & 0.16 & $60.24 \pm 22.91$ & $48.70 \pm 10.29$ & 0.35 \\
\hline Ki67 index & $13.67 \pm 21.64$ & $21.11 \pm 30.49$ & 0.47 & $31.75 \pm 30.06$ & $24.00 \pm 44.05$ & 0.69 \\
\hline $\begin{array}{l}\text { p-VEGFR2 (Y951) H } \\
\text { score }\end{array}$ & $36.47 \pm 44.99$ & $28.89 \pm 37.23$ & 0.67 & $57.08 \pm 43.82$ & $47.5 \pm 40.30$ & 0.7 \\
\hline $\begin{array}{l}\text { p-VEGFR2 (Y996) H } \\
\text { score }\end{array}$ & $8.24 \pm 9.00$ & $7.78 \pm 13.28$ & 0.91 & $34.17 \pm 46.16$ & $12.5 \pm 8.66$ & 0.37 \\
\hline \multicolumn{7}{|l|}{ Post-C1 } \\
\hline LVD & $0.41 \pm 0.54$ & $0.13 \pm 0.26$ & 0.16 & $0.85 \pm 1.25$ & $0.10 \pm 0.20$ & 0.26 \\
\hline VNI & $75.34 \pm 14.94$ & $68.91 \pm 24.67$ & 0.41 & $55.18 \pm 31.88$ & $74.63 \pm 5.07$ & 0.25 \\
\hline Ki67 index & $5.24 \pm 9.82$ & $7.89 \pm 12.94$ & 0.56 & $9.00 \pm 13.06$ & $27.5 \pm 34.03$ & 0.12 \\
\hline $\begin{array}{l}\text { p-VEGFR2 (Y951) H } \\
\text { score }\end{array}$ & $26.18 \pm 36.72$ & $25.56 \pm 35.04$ & 0.96 & $31.67 \pm 37.61$ & $25.00 \pm 43.58$ & 0.77 \\
\hline $\begin{array}{l}\text { p-VEGFR2 (Y996) H } \\
\text { score }\end{array}$ & $7.65 \pm 13.36$ & $2.22 \pm 4.41$ & 0.25 & $4 . .17 \pm 9.96$ & $7.50 \pm 15.50$ & 0.61 \\
\hline \multicolumn{7}{|l|}{$\begin{array}{l}\text { Post-C } 4^{\mathrm{b}} \\
(n=11 \text { for bevaci- } \\
\quad \text { zumab) }\end{array}$} \\
\hline LVD & $0.37 \pm 0.64$ & $0.40 \pm 0.55$ & 0.92 & $0.92 \pm 1.59$ & $0.20 \pm 0.34$ & 0.47 \\
\hline VNI & $80.66 \pm 12.64$ & $74.14 \pm 22.05$ & 0.34 & $71.13 \pm 29.80$ & $57.07 \pm 30.51$ & 0.5 \\
\hline Ki67 index & $2.71 \pm 9.62$ & $1.33 \pm 3.27$ & 0.68 & $7.5 \pm 10.35$ & $0.67 \pm 0.57$ & 0.29 \\
\hline $\begin{array}{l}\text { p-VEGFR2 (Y951) H } \\
\text { score }\end{array}$ & $32.67 \pm 43.70$ & $22.22 \pm 31.92$ & 0.53 & $43.75 \pm 48.67$ & $56.67 \pm 41.63$ & 0.69 \\
\hline $\begin{array}{l}\text { p-VEGFR2 (Y996) H } \\
\text { score }\end{array}$ & $8.53 \pm 15.48$ & $22.22 \pm 32.70$ & 0.15 & $7.50 \pm 11.67$ & $6.67 \pm 11.54$ & 0.91 \\
\hline
\end{tabular}

${ }^{a}$ Histological response could be analyzed in 26 of 29 patients in sunitinib cohort and 16 of 20 patients in bevacizumab cohort due to very little tissue remaining in block after prior sectioning for IHC analysis

${ }^{\mathrm{b}}$ In bevacizumab cohort, post-C4 biopsy IHC analysis was possible for 11 of total 16 patients. Of those 11 patients, 8 were good responders and 3 were poor responders

Cohort are concordant with our previous findings [11]. Somewhat surprisingly, these results were not replicated in the Bevacizumab Cohort in our current study. In order to tilt drug effects toward more vascularization normalization than anti-angiogenic, we used a sunitinib dose that was one-third full dose and administered it for only 5-7 days prior to each 2 -weekly cycle of chemotherapy instead of continuously. For bevacizumab, we used half dose $(5 \mathrm{mg} / \mathrm{kg}$ every 2 weeks rather than $10 \mathrm{mg} / \mathrm{kg}$ ) and administered it 1 week before chemotherapy rather than concurrently with chemotherapy. We postulate that the bevacizumab dose administered in our trial may still be too high, thus resulting in less prominent vascularization normalization effects than sunitinib.

The slight increase in LVD observed after bevacizumab treatment may be that bevacizumab largely sequesters VEGF-A, thereby blocking VEGF-A/VEGFR2 signaling. This may result in a compensatory increase in other
VEGF ligands like VEGF-C by tumor cells which then bind to VEGFR-3 on lymphatic endothelial cells leading to lymphangiogenesis [21]. On the other hand, the prominent effects on VNI and LVD seen with sunitinib can be on account of its action on multiple tyrosine kinase receptors. Apart from VEGFR, inhibition of other signaling pathways like platelet-derived growth factor receptor (PDGFR), stem cell factor receptor (KIT), FMS-like tyrosine kinase 3 (FLT3), colony-stimulating factor 1 receptor (CSF-1R), and rearranged during transfection (RET) may have resulted in the supplementary effect on tumor proliferation, angiogenesis and lymphangiogenesis [22, 23].

Breast tumors are known to produce VEGF and also express VEGFR2 on their surface. This autocrine signaling is responsible for tumor cell growth and division. VEGFR2 signaling can be inhibited by directly blocking the receptor or by interfering with the binding to its ligand VEGF. 
This is an anti-angiogenic effect and can be affected by both sunitinib, which blocks tyrosine phosphorylation of VEGFR, and bevacizumab which binds to and neutralizes VEGF. In a study on mouse mammary tumor model, sunitinib-treated mice showed decreased levels of tumor p-VEGFR2 [24]. We similarly observed decreased expression of tumor cell VEGFR2 after one cycle of chemotherapy in patients pretreated with sunitinib as well as bevacizumab proving that both drugs exert anti-angiogenic effects through inactivation of the VEGFR2 receptor on tumor cells. Intriguingly, p-VEGFR2 expression rebounded after 4 cycles of chemotherapy indicating that the action of anti-angiogenic agents in inhibiting the receptor activation on tumor cells could be of limited duration. Indeed, in an earlier phase $\mathrm{Ib}$ trial in breast cancer, we had observed sunitinib-induced normalization of tumor vasculature to occur as early as $24 \mathrm{~h}$; yet in another phase II randomized trial, intermittent, low-dose sunitinib combined with up to 6 cycles of docetaxel did not improve response rates compared to docetaxel alone [11]. We hypothesize that while initial treatment with sunitinib does normalize tumor vasculature, repeated administration may conversely compromise normal tumor vasculature and eventually impair chemotherapy delivery. In fact, it may be possible that just a single cycle or two of sunitinib prior to starting chemotherapy may be sufficient to normalize tumor vasculature [25]. On the other hand, the group that received bevacizumab showed a significant decrease in VEGFR2 expression on tumor cells in comparison to sunitinib. It is possible that the dose of the drugs could affect this autocrine loop signaling of tumor cells; a lower than clinically approved dose of sunitinib was used in this trial, while the bevacizumab dose administered was within the clinically approved range, with the latter thus exerting greater antiangiogenic than vasculature normalization effects. Also, it is possible that the primary action of sunitinib may have been on endothelial cells rather than tumor cells. Similar results have been reported in a study by Wedam et al., where bevacizumab was administered to locally advanced breast cancer patients $(n=21)$ and a significant inhibitory effect on tumor cell VEGFR2 expression (in both phosphorylation sites-Y951 and Y996) was demonstrated by IHC [26]. Collectively, these findings of decreased VEGFR2 expression together with lowered proliferation index suggests that both anti-angiogenic agents cause inactivation of VEGFR2 on tumor cells thus decreasing tumor cell proliferation although bevacizumab appears to exert a stronger effect than sunitinib on tumor cell VEGFR2 at the doses administered in this trial.

In the initial phase of study, both non-metastatic and metastatic patients were included, resulting in a small proportion (13\% in sunitinib cohort and $8 \%$ in bevacizumab cohort) of the study population having metastatic disease. Our data and conclusions remained unchanged when the analysis was performed on only non-metastatic patients. While we believe that our study of local immunohistochemical changes of vascular effects of low-dose anti-angiogenic effects on the breast tissue should be largely unaffected by the systemic effects of chemotherapy and disease burden in other disease sites, this is nonetheless a potential limitation of this study. In addition, while there is no single perfect method of evaluating tumor vessel normalization, histopathological analysis is considered one of the standard methods to successfully demonstrate the morphology and density of mature and immature vessels, although difficulty in vascular function assessment and dynamic monitoring of the vascularization process within the tumor is a pitfall [13, 27].

\section{Conclusion}

Immunohistochemistry analysis of serial tumor biopsies from patients with HER2-negative breast cancer who received lower dose sunitinib or bevacizumab before standard chemotherapy showed modulation of vessel morphology in tumor tissue along with suppression of tumor cell proliferation. Changes in LVD, VNI, Ki67 and p-VEGFR2, were generally early and observed after one cycle of treatment but tended to plateau with additional cycles of treatments. At the doses and schedule studied in this trial, sunitinib induced tumor vasculature normalization and inhibited lymphangiogenesis more prominently than bevacizumab, while bevacizumab demonstrated more significant effects on tumor VEGFR2 than sunitinib suggesting greater anti-angiogenic activity. Sunitinib with its more prominent vasculature normalization effects led to greater and sustained decline in tumor Ki67 than bevacizumab in this study, highlighting the promise of normalizing tumor vasculature to optimize chemotherapy delivery in breast cancer. The observation that vasculature normalization and anti-angiogenic effects plateaued or even rebounded with additional treatment cycles suggest that perhaps restricting the use of an anti-angiogenic agent to just the first one to two cycles of chemotherapy could be sufficient to exert the desired effects without the need to combine with all chemotherapy cycles. This strategy of more judicious combination of an anti-angiogenic agent with chemotherapy warrants further investigations.

Acknowledgements We are thankful to all patients who participated in the clinical trial.

Author contributions All authors contributed to at least one of the following: study conception, design, data acquisition, analysis, and/or interpretation. The first draft of the manuscript was written by KY and supervision/critical revision of the work was done by SCL. All authors have read and approved the final manuscript. 
Funding This research is supported by the Singapore Ministry of Health's National Medical Research Council under its Centre Grant (CG) Programme (CGAug16M005) and Clinician Scientist Award (SI Category, C/CSASI/0004/2015).

Data availability All data generated or analyzed during this current study are included in this published article.

\section{Declarations}

Conflict of interest SGWO served on advisory boards and had speaker's engagement with Pfizer, Novartis, Eli Lilly, AstraZeneca and Roche, and has received support to attend conference/travel from Pfizer, Novartis and AstraZeneca. AW has served on advisory board of Pfizer and Astra Zeneca. NN has received honoraria from Astra Zeneca, Janssen, Thermofisher and support to attend conferences from Astra Zeneca, Eisai. WQC has received conference sponsorship by MSD (ASCO 2021). GBC has been on advisory boards of MSD, AstraZeneca and Novartis and served as consultant to Adagene. He has received research support from BMS, MSD, Adagene and Taiho and has stock ownership of Merus and Gilead Sciences. SCL served on advisory boards and had speaker's engagement with Pfizer, Novartis, Eli Lilly, Astra Zeneca, Roche, and ACT genomics, received research grants/ grants to support clinical trials from Eisai, Taiho, Pfizer, and Karyopharm, and has received support to attend conference/travel from Amgen, Pfizer, Novartis, Roche and ACT Genomics.

Ethical approval This study was performed in line with the principles of the Declaration of Helsinki. Approval was granted by the National Healthcare Group Domain Specific Ethics Review Board in 2016 (DSRB 2016/00327).

Informed consent Signed written informed consent was obtained from all individual participants included in the study.

Open Access This article is licensed under a Creative Commons Attribution 4.0 International License, which permits use, sharing, adaptation, distribution and reproduction in any medium or format, as long as you give appropriate credit to the original author(s) and the source, provide a link to the Creative Commons licence, and indicate if changes were made. The images or other third party material in this article are included in the article's Creative Commons licence, unless indicated otherwise in a credit line to the material. If material is not included in the article's Creative Commons licence and your intended use is not permitted by statutory regulation or exceeds the permitted use, you will need to obtain permission directly from the copyright holder. To view a copy of this licence, visit http://creativecommons.org/licenses/by/4.0/.

\section{References}

1. Bray F, Ferlay J, Soerjomataram I et al (2018) Global cancer statistics 2018: GLOBOCAN estimates of incidence and mortality worldwide for 36 cancers in 185 countries. CA: Cancer J Clin 68:394-424. https://doi.org/10.3322/caac. 21492

2. Schneider BP, Miller KD (2005) Angiogenesis of breast cancer. J Clin Oncol 23:1782-1790. https://doi.org/10.1200/JCO.2005.12. 017

3. Fox SB, Generali DG, Harris AL (2007) Breast tumour angiogenesis. Breast Cancer Res 9:216. https://doi.org/10.1186/bcr1796
4. Bear HD, Tang G, Rastogi P et al (2012) Bevacizumab added to neoadjuvant chemotherapy for breast cancer. N Engl J Med 366:310-320. https://doi.org/10.1056/NEJMoa1111097

5. Rugo HS (2012) Inhibiting angiogenesis in breast cancer: the beginning of the end or the end of the beginning? J Clin Oncol 30:898-901. https://doi.org/10.1200/JCO.2011.38.5492

6. Maj E, Papiernik D, Wietrzyk J (2016) Antiangiogenic cancer treatment: the great discovery and greater complexity (Review). Int J Oncol 49:1773-1784. https://doi.org/10.3892/ijo.2016.3709

7. Jain RK (2005) Normalization of tumor vasculature: an emerging concept in antiangiogenic therapy. Science 307:58-62. https://doi. org/10.1126/science. 1104819

8. Perrot-Applanat M, Di Benedetto M (2012) Autocrine functions of VEGF in breast tumor cells. Cell Adh Migr 6:547-553. https:// doi.org/10.4161/cam.23332

9. Wolff AC, Hammond MEH, Allison KH et al (2018) Human epidermal growth factor receptor 2 testing in breast cancer: American Society of Clinical Oncology/College of American Pathologists Clinical Practice Guideline Focused Update. Arch Pathol Lab Med 142:1364-1382. https://doi.org/10.5858/arpa.2018-0902-SA

10. Gaustad JV, Simonsen TG, Smistad R et al (2015) Early effects of low dose bevacizumab treatment assessed by magnetic resonance imaging. BMC Cancer. https://doi.org/10.1186/ s12885-015-1918-1

11. Wong ALA, Sundar R, Wang TT et al (2016) Phase Ib/II randomized, open-label study of doxorubicin and cyclophosphamide with or without low-dose, short-course sunitinib in the pre-operative treatment of breast cancer. Oncotarget. https:// doi.org/10.18632/oncotarget.11596

12. Therasse P, Arbuck SG, Eisenhauer EA et al (2000) New guidelines to evaluate the response to treatment in solid tumors. JNCI J Natl Cancer Inst 92:205-216. https://doi.org/10.1093/jnci/ 92.3.205

13. Fakhrejahani E, Toi M (2012) Tumor angiogenesis: pericytes and maturation are not to be ignored. J Oncol 2012:1-10. https://doi.org/10.1155/2012/261750

14. Trinh XB, Tjalma WAA, Vermeulen PB et al (2009) The VEGF pathway and the AKT/mTOR/p70S6K1 signalling pathway in human epithelial ovarian cancer. Br J Cancer 100:971-978. https://doi.org/10.1038/sj.bjc.6604921

15. Dowsett M, Smith IE, Ebbs SR et al (2007) Prognostic value of Ki67 expression after short-term presurgical endocrine therapy for primary breast cancer. J Natl Cancer Inst. https://doi.org/10. 1093/jnci/djk020

16. Smith IC (2002) Neoadjuvant chemotherapy in breast cancer: significantly enhanced response with docetaxel. J Clin Oncol 20:1456-1466. https://doi.org/10.1200/JCO.20.6.1456

17. Tong RT, Boucher Y, Kozin SV et al (2004) Vascular normalization by vascular endothelial growth factor receptor 2 blockade induces a pressure gradient across the vasculature and improves drug penetration in tumors. Can Res 64:3731-3736. https://doi. org/10.1158/0008-5472.CAN-04-0074

18. Winkler F, Kozin SV, Tong RT et al (2004) Kinetics of vascular normalization by VEGFR2 blockade governs brain tumor response to radiation. Cancer Cell 6:553-563. https://doi.org/ 10.1016/j.ccr.2004.10.011

19. Robert NJ, Diéras V, Glaspy J et al (2011) RIBBON-1: Randomized, double-blind, placebo-controlled, phase III trial of chemotherapy with or without bevacizumab for first-line treatment of human epidermal growth factor receptor 2-negative, locally recurrent or metastatic breast cancer. J Clin Oncol. https://doi.org/10.1200/JCO.2010.28.0982

20. El-Gohary YM, Metwally G, Saad RS et al (2008) Prognostic significance of intratumoral and peritumoral lymphatic density and blood vessel density in invasive breast carcinomas. Am 
J Clin Pathol 129:578-586. https://doi.org/10.1309/2HGNJ 1 GU57JMBJAQ

21. Michaelsen SR, Staberg M, Pedersen H et al (2018) VEGF-C sustains VEGFR2 activation under bevacizumab therapy and promotes glioblastoma maintenance. Neuro Oncol 20:14621474. https://doi.org/10.1093/neuonc/noy 103

22. Zhao Y, Adjei AA (2015) Targeting angiogenesis in cancer therapy: moving beyond vascular endothelial growth factor. Oncologist 20:660-673. https://doi.org/10.1634/theoncologist. 2014-0465

23. Aparicio-Gallego G, Blanco M, Figueroa A et al (2011) New insights into molecular mechanisms of sunitinib-associated side effects. Mol Cancer Ther 10:2215-2223. https://doi.org/10.1158/ 1535-7163.MCT-10-1124

24. Tanaka Y, Shibata MA, Morimoto J, Otsuki Y (2011) Sunitinib suppresses tumor growth and metastases in a highly metastatic mouse mammary cancer model. Anticancer Res 31:1225

25. Ang YLE, Ho GF, Soo RA et al (2020) A randomized phase II trial evaluating the addition of low dose, short course sunitinib to docetaxel in advanced solid tumours. BMC Cancer 20:1118. https://doi.org/10.1186/s12885-020-07616-4

26. Wedam SB, Low JA, Yang SX et al (2006) Antiangiogenic and antitumor effects of bevacizumab in patients with inflammatory and locally advanced breast cancer. J Clin Oncol 24:769-777. https://doi.org/10.1200/JCO.2005.03.4645

27. Li W, Quan YY, Li Y et al (2018) Monitoring of tumor vascular normalization: the key points from basic research to clinical application. Cancer Manag Res 10:4163-4172. https://doi.org/10.2147/ CMAR.S174712

Publisher's Note Springer Nature remains neutral with regard to jurisdictional claims in published maps and institutional affiliations. 